\title{
No effect of albumin infusion on the prevention of hepatic encephalopathy after transjugular intrahepatic portosystemic shunt
}

\author{
Oliviero Riggio $^{1,2}$ - Silvia Nardelli ${ }^{1}$ - Chiara Pasquale ${ }^{1}$ - Ilaria Pentassuglio ${ }^{1}$. \\ Stefania Gioia ${ }^{1}$. Eugenia Onori ${ }^{1}$. Camilla Frieri ${ }^{1} \cdot$ Filippo Maria Salvatori $^{1}$. \\ Manuela Merli ${ }^{1}$
}

Received: 16 March 2015 / Accepted: 16 July 2015 / Published online: 20 August 2015

(C) European Union 2015

\begin{abstract}
Hepatic encephalopathy (HE) is a major problem in patients submitted to TIPS. Previous studies identified low albumin as a factor associated to post-TIPS HE. In cirrhotics with diuretic-induced HE and hypovolemia, albumin infusion reduced plasma ammonia and improved HE. Our aim was to evaluate if the incidence of overt HE (grade II or more according to $\mathrm{WH}$ ) and the modifications of venous blood ammonia and psychometric tests during the first month after TIPS can be prevented by albumin infusion. Twenty-three patients consecutively submitted to TIPS were enrolled and treated with $1 \mathrm{~g} / \mathrm{Kg} \mathrm{BW}$ of albumin for the first 2 days after TIPS followed by $0,5 \mathrm{~g} / \mathrm{Kg} \mathrm{BW}$ at day 4 th and 7 th and then once a week for 3 weeks. Forty-five patients included in a previous RCT (Riggio et al. 2010) followed with the same protocol and submitted to no pharmacological treatment for the prevention of $\mathrm{HE}$,
\end{abstract}

Key points - hepatic encephalopathy (HE) is very frequent in patients submitted to TIPS

- A previous study (Riggio et al. 2008) identified high creatinine levels low serum sodium and albumin as associated to post TIPS HE, suggesting that the correction of hypovolemia might be useful in HE prevention

- In our study no differences in the incidence of overt HE were observed between the group of patients treated with albumin and historical controls during the first month ( 34 vs $31 \%$ ) or during the follow-up (39 vs $48 \%$ ). Thus albumin infusion has not a role in the prevention of HE after a TIPS.

Oliviero Riggio

oliviero.riggio@uniroma1.it

1 Department of Clinical Medicine, Center for the Diagnosis and Treatment of Portal Hypertension "Sapienza" University of Rome, Rome, Italy

2 Centro di Riferimento per l'Ipertensione Portale, II Gastroenterologia, Dipartimento di Medicina Clinica, "Sapienza" Università di Roma, Viale dell'Università 37, 00185 Roma, Italy were used as historical controls. No differences in the incidence of overt HE were observed between the group of patients treated with albumin and historical controls during the first month (34 vs $31 \%$ ) or during the follow-up ( 39 vs $48 \%$ ). Two patients in the albumin group and three in historical controls needed the reduction of the stent diameter for persistent HE. Venous blood ammonia levels and psychometric tests were also similarly modified in the two groups. Survival was also similar. Albumin infusion has not a role in the prevention of post-TIPS HE.

Keywords TIPS · Hepatic encephalopathy $\cdot$ Liver cirrhosis · Albumin

\author{
Abbreviations \\ TIPS Transjugular intrahepatic portosystemic shunt \\ DST Digit-symbol test \\ TMT-A Trail-making test A \\ TMT-B Trail-making test B
}

\section{Introduction}

Transjugular intrahepatic portosystemic shunt (TIPS) is currently used for the treatment of the complications of portal hypertension (Cabrera et al. 1996; Sanjal et al. 1996; Rossle et al. 1997, 2000; Merli et al. 1998; Lebrec et al. 1996; Gines et al. 2002; Sanyal et al. 2003; Salerno et al. 2004). This procedure, however, involves two main drawbacks: the occurrence of shunt dysfunction (Cabrera et al. 1996; Merli et al. 1998; Lind et al. 1994; LaBerge et al. 1995; Nazarian et al. 1994; Latimer et al. 1998) and of hepatic encephalopathy (HE) which is very frequent 
after a TIPS (Sanjal et al. 1994; Jalan et al. 1995; Somberg et al. 1995; Riggio et al. 1996; Zuckerman et al. 1997; Nolte et al. 1998). Moreover, post TIPS HE refractory to standard treatments may occur in about $8 \%$ of patients (Riggio et al. 2008). Unfortunately, until now, no attempt aimed at reducing the incidence of post-TIPS $\mathrm{HE}$ has been successful. A RCT failed to show an effect of the administration of non-absorbable disaccharides or antibiotics on the incidence of HE during the first month after TIPS procedure (Riggio et al. 2005). Moreover, the use of stents with lower diameter ( 8 versus $10 \mathrm{~mm}$ ) failed to reduce the incidence of post TIPS HE and resulted less efficient in controlling the complications of portal hypertension (Riggio et al. 2010).

A previous study (Riggio et al. 2008) on the incidence of HE in patients submitted to TIPS with PTFE covered stents identified older age, high creatinine levels, low serum sodium and albumin levels as independent factors associated to post TIPS HE, suggesting that hypovolemia may be involved in the pathogenesis of this complication and that the correction of hypovolemia might be useful in its prevention. The effect of albumin in patients submitted to TIPS has never been tested, but in a small clinical trial (Rajiv and Dharmesh 2004), albumin infusion was able to ameliorate HE and to increase urinary ammonia excretion on cirrhotic patients with diuretic-induced HE. In that study, albumin was more efficient then other plasma expander suggesting that albumin, in addition to its plasma expander capacity may have other potential effects. Albumin is able to bind different molecules (fatty acids, bilirubin, bile salts, amino acids and nitric oxide), to influence the redox balance by reducing oxidative stress and to reduce brain swelling in a model of ischemic stroke in experimental animals (Belayev et al. 2001). Based on the above considerations, albumin infusion was tested for the treatment of overt $\mathrm{HE}$ in cirrhotic patients in a recent RCT (Simón-Talero et al. 2013). Moreover, TIPS placement induces a complex modification of systemic hemodynamic resulting in increased cardiac output, decreased peripheral vascular resistance and increase of cerebral blood flow (Kavitt et al. 2008). This increment may amplify the availably of ammonia to the brain and facilitate the astrocytes swelling, a condition which may be facilitated by a pre-existing hyponatremia. Thus, a treatment to reduce brain swelling may be beneficial.

The above considerations encouraged us in testing the efficacy of albumin infusion in the prevention of HE after TIPS in a pilot study aimed at eventually supporting the proposal of a randomized controlled trial. We compared the incidence of post TIPS HE observed in this pilot study to that observed in a previous randomized control trial designed for the prevention of post TIPS HE (Riggio et al. 2010).

\section{Patients and methods}

All consecutive cirrhotics admitted to our Gastroenterology Unit to be submitted to TIPS were enrolled. Exclusion criteria corresponded to the contraindications to TIPS used in our centre: past or present history of recurrent HE, serious cardiac or pulmonary dysfunction, diagnosis of hepatocellular carcinoma, sepsis.

The purpose of the study, the enrolment, the follow-up and the details of TIPS operation were explained to the patients and a written informed consent was obtained. The study protocol conformed to the ethical guidelines of the 1975 Declaration of Helsinki and was approved by the Ethical and the Research Committees of the "Sapienza" University of Rome. The study was registered in Clinicaltrials.gov with the number NCT01559519.

All patients were hospitalized before TIPS placement. At enrolment the patients' medical histories were collected; physical examinations, calculations of Child-Pugh's and MELD scores, doppler ultrasonography and upper gastrointestinal endoscopy were carried out. TIPS procedures were performed by the same radiology team as previously described. (Merli et al. 1998; Sampietro et al. 1998; Angeloni et al. 2004; Rossi et al. 2004). Endoscopy was repeated 1 week after TIPS. The patients remained in hospital for at least 7 days after TIPS placement and were successively seen weekly during the first month. Ultrasonography was repeated weekly to exclude shunt dysfunction. Shunt patency was assessed with colorDoppler, measurement of the mean flow velocity and direction within the shunt at three sites (proximal, medial and distal portion), in the portal vein and in intrahepatic portal branches. The diameters of the stent, the portal vein and the spleen were also measured. All the subjects were evaluated and followedup by the same medical team by a prospective protocol diagnostic work-up and a surveillance strategy which was identical to that used in a previously published RCT which provided the historical controls (Rajiv and Dharmesh 2004).

Patients were treated with albumin infusion according to the following scheme: $1 \mathrm{~g} / \mathrm{Kg}$ body weight for the first 2 days after TIPS followed by $0,5 \mathrm{~g} / \mathrm{Kg}$ body weight at day 4 and then $0,5 \mathrm{~g} / \mathrm{Kg}$ body weight once a week for the remaining 3 weeks.

The albumin (Human Albumin $20 \%$ solution) used for the study was kindly supported by GORE srl which is the producer of the PTFE covered stents used in our university hospital (Viatorr®, W.L. Gore \& Associates, Inc., Flagstaff, AZ, USA). GORE was not involved in the conception, protocol, analysis of the results and discussion of the study.

\section{Evaluation of $\mathrm{HE}$}

The day before the procedure, a basal evaluation of HE including examination and grading of the patients' mental state, 
asterixis, and psychometric performance [TMT-A and TMTB; Digit Symbol, (DS)] as well as their venous blood ammonia determination were carried out. The evaluation of the degree of HE was based on the alteration of the patient's mental state using modifications of the West Haven Criteria (Conn et al. 1977). Blood samples from a peripheral vein were collected into iced tubes for ammonia determination, performed immediately after using the Ammonia Checker II (Menarini, Florence, Italy), as previously described (Nicolao et al. 2003). This workout (evaluation of mental status, psychometric performance and venous blood ammonia determination) was repeated at weekly intervals during the first month after the TIPS procedure. TMT-A, TMT-B, and DS results were expressed as Z-score, adjusted for the main confounders (age and education) according to Amodio et al. (Amodio et al. 2002). Both the patients and their families were instructed about the importance of an immediate contact with the medical staff should any alteration in the mental state occur in between the scheduled visits. In particular, the family was instructed to refer to the occurrence of lethargy, apathy, obvious personality change, inappropriate behaviour, or disorientation for time and place (corresponding to a grade-II alteration of the patients' mental state). In this case, the HE evaluation, including the psychometric performance, was repeated to confirm and stage the degree of HE. None of the patients received any pharmacological treatment to prevent the occurrence of HE, in particular lactulose or rifaximin were avoided. Once developed, HE was treated with oral administration of non-absorbable disaccharides or non-absorbable antibiotics. All potential HE precipitating events were treated.

\section{End-points}

The primary end-point of the study was the incidence of overt HE (grade II or higher grades) in the first month after TIPS placement. All the other episodes of HE occurring during the follow-up were also recorded. Other outcomes of interest were: the modification of blood ammonia and of the psychometric performance 1 month after TIPS; the occurrence of refractory $\mathrm{HE}$ defined as recurrent $\mathrm{HE}$ (at least three episodes of non-precipitant-induced $\mathrm{HE}$ requiring hospitalization in the last 3 months despite continuous treatment with lactulose) or persistent HE (presence of continuously detectable altered mental state with further episodic deterioration despite protein restriction and treatment with lactulose) and survival.

\section{Statistical analysis}

Results are expressed as "mean \pm SD". Comparisons to historical data from a previously published RCT (Riggio et al. 2010) were included, based on methodology and recommendations from regulatory guidance and published literature (Gehan 1984; Pocock 1976; Food and Drug Administration Center for Drug Evaluation and Research (CDER) Center for Biologics Evaluation and Research (CBER) 2001). In this historical RCT, enrolment criteria, patients 'evaluation and followup as well as clinical end-points were similar to those adopted in the present study. Moreover, the two studies were supported by the same organization and investigators.

Comparisons among the groups were performed for quantitative data by the Student's $t$-test. The chi-squared test was used to determine the differences in proportions. The incidence of HE as well as of the other outcomes of the study, including mortality was calculated by the Kaplan-Meier method (Kaplan and Meier 1958) and compared by the logrank test. Follow-up was measured in months from the date of successful TIPS procedure until death, liver transplantation, or the most recent clinical examination. Patients were considered lost to follow-up if they did not show up at two consecutive 6month clinical examinations. The SPSS program was used for all computations.

\section{Sample size calculation}

On a previous study on the pharmacological prophylaxis of post-TIPS HE (Riggio et al. 2005) the incidence of HE during the first month after TIPS was $33 \%$, (95 \% CI 22-45\%). It is thus estimated that a group of 20 patients would be required to observe a reduction of this rate to about $10 \%$.

\section{Results}

The demographic, clinical and biochemical characteristics of the patients are reported in Table 1. For comparison, the same parameters observed in the historical controls are also reported. There were no significant differences between the two groups in clinical and laboratory data at the time of TIPS placement. Both groups were comparable for age, gender, aetiology and severity of liver disease including Child-Pugh and MELD scores. They also had a similar history of previous ascites and $\mathrm{HE}$ and did not differ concerning the size of varices and the indications for TIPS. TIPSs were successfully implanted in all patients. After TIPS placement, the portosystemic pressure gradient significantly decreased in both groups (from $18.6 \pm 5.2$ to $5.4 \pm 3.6 \mathrm{mmHg}$ in the albumin group and from $22.3 \pm 6.7$ to $7.1 \pm 3.9 \mathrm{mmHg}$ in the historical control group; $p<0.001$ in both groups). During the first month after TIPS, HE occurred in 8 of the 23 patients (34\%) in the albumin group. HE occurred spontaneously in three patients, while infection was the precipitating event in the remaining five patients. The eight patients with overt $\mathrm{HE}$ during the first month of follow up were older (64 \pm 3 vs $54 \pm$ 11 years; $p=0.01)$ and with higher TMT-B (147 \pm 45 vs $98 \pm$ $41 \mathrm{~s} . ; p=0.04)$ than the patients without overt HE. During the 
Table 1 Demographic and clinical characteristics of the patients included in the study and the historical controls (Riggio O. et al. J Hepatol. 2010)

\begin{tabular}{|c|c|c|c|}
\hline & $\begin{array}{l}\text { Albumin } \\
(n=23)\end{array}$ & $\begin{array}{l}\text { Historical } \\
\text { Controls }(n=45)\end{array}$ & $P$ Value \\
\hline $\operatorname{Sex}(M / F)$ & $17 / 6$ & $28 / 17$ & Ns \\
\hline Age (yrs.) & $57.7 \pm 10$ & $55.2 \pm 10.7$ & Ns \\
\hline $\begin{array}{l}\text { Etiology (virus/ } \\
\text { alcohol/other) }\end{array}$ & $9 / 8 / 6$ & $18 / 18 / 9$ & Ns \\
\hline MELD & $11.5 \pm 3.3$ & $10.4 \pm 4.2$ & Ns \\
\hline $\begin{array}{l}\text { Child Pugh class } \\
\text { (A/B/C) }\end{array}$ & $7 / 14 / 2$ & $10 / 25 / 10$ & Ns \\
\hline $\begin{array}{l}\text { TIPS indication } \\
\text { (bleeding/ } \\
\text { refractory ascites) }\end{array}$ & $13 / 10$ & $21 / 24$ & Ns \\
\hline $\begin{array}{l}\text { Previous HE n. } \\
\text { of pts. }(\%)\end{array}$ & $0(0 \%)$ & $9(20 \%)$ & 0.02 \\
\hline Bilirubin (mg/dl) & $1.5 \pm 1.4$ & $1.7 \pm 1.4$ & Ns \\
\hline Albumin (g/dl) & $3.3 \pm 0.6$ & $3.4 \pm 0.5$ & Ns \\
\hline Sodium $(\mathrm{mEq} / \mathrm{L})$ & $136 \pm 5$ & $137.4 \pm 4.9$ & Ns \\
\hline $\begin{array}{l}\text { Portosystemic Pressure } \\
\text { Gradient }(\mathrm{mmHg}) \\
\text { pre-TIPS }\end{array}$ & $18.6 \pm 5$ & $22.3 \pm 6.7$ & 0.04 \\
\hline $\begin{array}{l}\text { Portosystemic Pressure } \\
\text { Gradient }(\mathrm{mmHg}) \\
\text { after TIPS }\end{array}$ & $5.4 \pm 3.6$ & $7.1 \pm 3.6$ & Ns \\
\hline $\begin{array}{l}\text { Venous ammonia } \\
\text { level }(\mu \mathrm{g} / \mathrm{dl})\end{array}$ & $41 \pm 15$ & $48 \pm 31$ & Ns \\
\hline TMT-A (sec) & $58 \pm 25$ & $60 \pm 40$ & Ns \\
\hline TMT-B (sec) & $116 \pm 55$ & $110 \pm 62$ & Ns \\
\hline DS (n) & $23 \pm 9$ & $31 \pm 13$ & 0.01 \\
\hline Mean follow-up (months) & $6.6 \pm 4.8$ & $8.6 \pm 9$ & Ns \\
\hline
\end{tabular}

first month after TIPS, overt HE occurred in 14 of the 45 patients (31\%) in the control group.

Three patients in the historical control group and two patients in the study group were lost to follow-up. These patients were included in the analysis and censored at the time of the last visit. The cumulative incidence of HE during the follow up was not statistically different in the patients treated with albumin and in the historical controls (Fig. 1). Two of the 23 patients treated with albumin suffered of a refractory HE ( $8 \%$ ), which did not respond to the medical therapy. In one patient chronic HE started within the first month after TIPS. In these two patients the cognitive impairment improved after the reduction of the calibre of the stent. The porto-systemic pressure gradient before the diameter reduction was 11 and $9.8 \mathrm{mmHg}$, respectively. Three of the 45 patients among the historical controls experienced refractory HE (7\%). At baseline there were no significant differences between the two groups in the venous ammonia levels and in TMT-A and TMT-B. DS test was slightly better in the control group (Table 1). After TIPS placement, venous ammonia levels

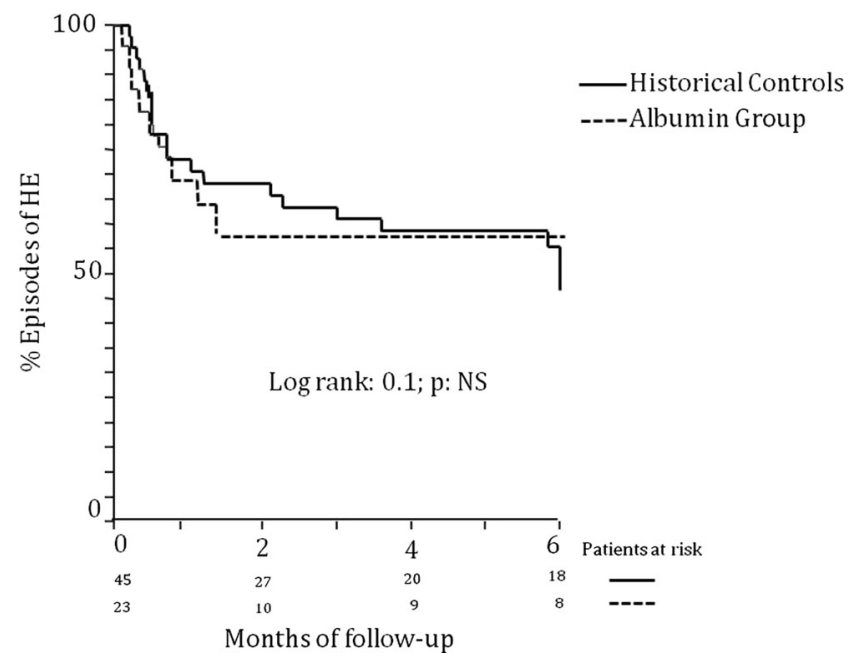

Fig. 1 Cumulative incidence of Hepatic Encephalopathy during the follow-up in the patients treated with albumin and in the historical controls

increased significantly in both groups. The psychometric performance was unmodified after TIPS (Table 2).

Five patients in albumin group $(22 \%)$ died during the follow-up. Deaths were due to an accident in one patient, to a stroke in another one and to liver failure in the other patients. Eight patients in historical control group (18\%) died during follow up (Fig. 2).

\section{Discussion}

The present study failed to show that the volume espansion induced by the infusion of albumin was able to reduce the incidence of overt HE after TIPS. In fact, compared to the hystorical controls, the cumulative incidence of overt $\mathrm{HE}$

Table 2 Comparison of psychometric tests and venous ammonia levels before and 1 month after TIPS placement

\begin{tabular}{llll}
\hline Albumin group (17 pts) & & & \\
& Pre-TIPS & After TIPS & $P$ Value \\
TMT-A (sec) & $59.1 \pm 28.6$ & $64.6 \pm 35.6$ & $\mathrm{Ns}$ \\
TMT-B (sec) & $116.4 \pm 62.6$ & $138.6 \pm 95.2$ & $\mathrm{Ns}$ \\
DS (n $\left.{ }^{\circ}\right)$ & $24.5 \pm 9.8$ & $21.8 \pm 10.9$ & $\mathrm{Ns}$ \\
Venous ammonia $(\mu \mathrm{g} / \mathrm{dl})$ & $40.7 \pm 12.2$ & $89 \pm 32$ & 0.001 \\
Historical controls $(31 \mathrm{pts})$ & & & \\
& Pre-TIPS & After TIPS & $P$ Value \\
TMT-A (sec) & $55.6 \pm 38.7$ & $51.2 \pm 34.5$ & $\mathrm{Ns}$ \\
TMT-B (sec) & $99.2 \pm 48.7$ & $117.1 \pm 79.6$ & $\mathrm{Ns}$ \\
DS (n $\left.{ }^{\circ}\right)$ & $33.7 \pm 12$ & $34.7 \pm 14.6$ & $\mathrm{Ns}$ \\
Venous ammonia $(\mu \mathrm{g} / \mathrm{dl})$ & $47.6 \pm 36.6$ & $89.7 \pm 46.6$ & 0.001 \\
\hline
\end{tabular}

Paired $t$ test 


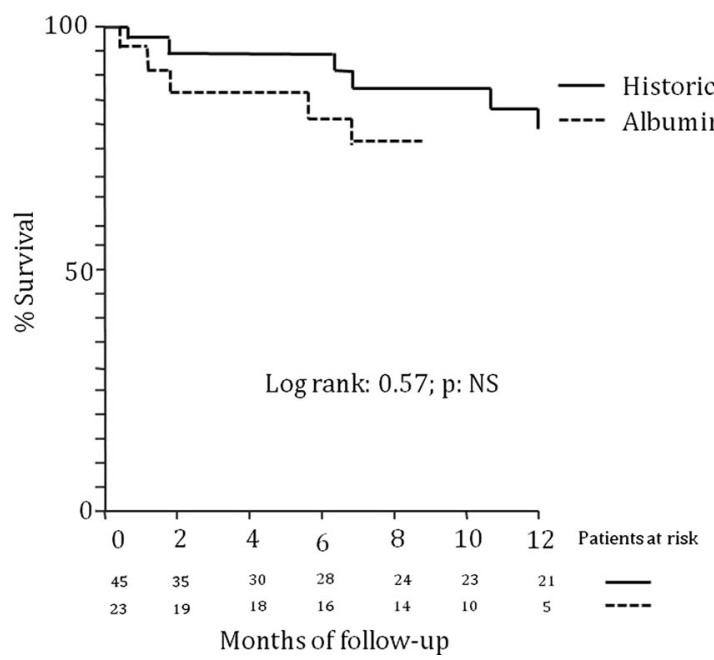

Fig. 2 Survival in patients in the patients treated with albumin and in the historical controls

was similar both during the first month and during the whole follow up. Moreover, the number of patients with persistent HE refractory to treatment and necessitating the reduction of the stent diameter was also similar as well as the modifications of venous ammonia and psycometric tests 1 month after TIPS. Although, based on the result of a pilot study with hystorical controls and not of a randomised controlled trial, we conclude that albumin infusion has no role in the prevention of postTIPS HE.

The choice of carring on a pilot study instead of a RCT was based on different considerations. First, the possible role of albumin in the prevention of HE after TIPS was postulated more on the basis of a statistical association than on pathophysiological considerations. Hypoalbuminemia was found to be a risk factor for HE after TIPS in two studies [16; 20]. In the latter, in common with albumin, also high creatinine levels and low serum sodium concentration were independently associated to post TIPS HE, suggesting a role for hypovolemia in the pathogenesis of this complication. Hypovolemia may increase plasma ammonia levels by reducing urinary ammonia excretion or by inducing circulatory abnormalities including changes in cerebral blood flow and metabolism (Iversen et al. 2009). Actually, volume expansion induced by saline infusion resulted in a reduction in plasma ammonia concentrations and an increase in urinary ammonia excretion in 16 well compensated cirrhotic patients without HE (Jalan and Kapoor 2003). Moreover, albumin infusion was able to ameliorate HE and to increase urinary ammonia excretion in eight cirrhotic patients with diuretic-induced HE. Another possible role of albumin in HE treatment derives from its scavenger action.

On the other hand, we are confident that our conclusion based on the comparison between the study group treated with albumin and the hystorical controls are quite reliable. In fact, our hystorical control group included patients enrolled in a RCT (Riggio et al. 2010) previously carried on with the same inclusion criteria of this study. In particular, both the patients included in the study group and the historical controls were enrolled, operated on and prospectively followed-up by the same investigators by using the same methodology to establish the incidence of $\mathrm{HE}$ as well as the modification cognitive fuction and plasma ammonia after TIPS. Thus the present study is based on methodology and recommendations from regulatory guidance and published literature for the use of hystorical controls (Gehan 1984; Pocock 1976; Food and Drug Administration Center for Drug Evaluation and Research (CDER) Center for Biologics Evaluation and Research (CBER) 2001).

The possibility that our negative results could depend on the administration of insufficient amount of albumin, may not be completely ruled out. We used $1 \mathrm{~g} / \mathrm{Kg}$ body weight for the first 2 days after TIPS followed by $0,5 \mathrm{~g} / \mathrm{Kg}$ body weight at day 4 and then by $0,5 \mathrm{~g} / \mathrm{Kg}$ body weight once a week for 3 weeks to obtain a plasma expansion immediately after TIPS and for the following 4 weeks. This amount was chosen because in cirrhotic patients, the dose used to obtain a significant blood volume expansion was 1,5 and $1,0 \mathrm{~g} / \mathrm{kg}$ BW at day 1 and 3 respectively in patients affected by spontaneous bacterial peritonitis (Sort et al. 1999) and 1,0 g/kg BW for 2 days in patients with suspected hepato-renal syndrome (Salerno et al. 2007). In experimental animals with ischemic stroke, the infusion of $0.7-1.25 \mathrm{~g} / \mathrm{kg} \mathrm{BW}$ of albumin was able to obtain a reduction in brain swelling. Finally, the maintenance dose of $0.5 \mathrm{~g}$ of albumin per $\mathrm{Kg} \mathrm{BW}$, every week for further 3 weeks was based on practical considerations.

Another point to be underlined derives from the observation that an infection was identified and considered as the precipitating event in 5 out of the 8 patients who experienced HE during the first month after TIPS. Thus, only three patients had a spontaneous HE which may directly linked to the opening of the porto-systemic shunt. This was not the experience in our historical controls, in whom post TIPS HE was spontaneous in all cases. Thus, on this point, the methodology used (pilot study and not RCT) may be relevant. Finally, in a recently published RCT albumin infusion failed to improve the resolution of HE during hospitalization (Simón-Talero et al. 2013) suggesting that albumin does not have a major impact on HE in cirrhotics that develop an episode of HE.

Our results are in keeping with this observation and suggest that albumin has no impact prevention of HE after TIPS.

Acknowledgments The study was partially supported by W.L. GORE and Associated which is the producer of the stents used in the study for TIPS costruction.

Conflict of interest The authors declare that no conflict of interest exists concerning this paper. 
Author contributions Oliviero Riggio: study concept and design, analysis and interpretation of data; manuscript preparation; final drafting of the manuscript; study supervision.

Silvia Nardelli: acquisition and analysis of data.

Chiara Pasquale: acquisition and analysis of data.

Ilaria Pentassuglio: acquisition of data.

Stefania Gioia: acquisition of data

Eugenia Onori: acquisition of data

Camilla Frieri: acquisition of data

Manuela Merli: critical discussion and support, manuscript revision for important intellectual content.

\section{References}

Amodio P, Wenin H, Del Piccolo F et al (2002) Variability of trail making test, symbol digit test and line trait test in normal people. A normative study taking into account age-dependent decline and sociobiological variables. Aging Clin Exp Res 14:117-131

Angeloni S, Merli M, Salvatori F et al (2004) Polytetrafluoroethylenecovered stentgraf for TIPS procedure: 1-year patency and clinical results. Am J Gastroenterol 99:280-285

Belayev L, Liu Y, Zhao W et al (2001) Human albumin therapy of acute ischemic stroke: marked neuroprotective efficacy at moderate doses and with a broad therapeutic window. Stroke 32:553-560

Cabrera J, Maynar M, Granados R et al (1996) Transjugular intrahepatic portosystemic shunt versus sclerotherapy in the elective treatment of variceal hemorrhage. Gastroenterology 110:832-839

Conn HO, Leevy CM, Vlahcevic ZR et al (1977) Comparison of lactulose and neomycin in the treatment of chronic portal-systemic encephalopathy. A double blind controlled trial. Gastroenterology 72:573583

Food and Drug Administration Center for Drug Evaluation and Research (CDER) Center for Biologics Evaluation and Research (CBER) (2001) Guidance for industry: E 10: choice of control group and related issues in clinical trials. US Food and Drug Administration Center for Biologics Evaluation and Research, Rockville

Gehan EA (1984) The evaluation of therapies: historical control studies. Stat Med 3:315-324

Gines P, Uriz J, Calahorra B et al (2002) Transjugular intrahepatic portosystemic shunt versus paracentesis plus albumin for refractory ascites in cirrhosis. Gastroenterology 123:1839-1847

Iversen P, Sorensen M, Bak LK et al (2009) Low cerebral oxygen consumption and blood flow in patients with cirrhosis and an acute episode of hepatic encephalopathy. Gastroenterology 136:863-871

Jalan R, Kapoor D (2003) Enhanced renal ammonia excretion following volume expansion in patients with well compensated cirrhosis of the liver. Gut 52:1041-1045

Jalan R, Elton RA, Redhead DN et al (1995) Analysis of prognostic variables in the prediction of mortality, shunt failure, variceal rebleeding and encephalopathy following the transjugular intrahepatic portosystemic shunt for variceal haemorrhage. J Hepatol 23:123-128

Kaplan GL, Meier P (1958) Non-parametric estimation from incomplete observations. J Am Stat Assoc 53:457-481

Kavitt RT, Yang VL, Jensen DM (2008) Cerebral edema and hyperammonemia after transjugular intrahepatic portosystemic shunt placement in a cirrhotic patient. Clin Gastroenterol Hepatol 6:1054-1056

LaBerge JM, Somberg KA, Lake JR et al (1995) Two-year outcome following transjugular intrahepatic portosystemic shunt for variceal bleeding: results in 90 patients. Gastroenterology 108:1143-1151
Latimer J, Bawa SM, Rees CJ et al (1998) Patency and reintervention rates during routine TIPSS surveillance. Cardiovasc Intervent Radiol 21:234-239

Lebrec D, Giuily N, Hadengue A et al (1996) Transjugular intrahepatic portosystemic shunts: comparison with paracentesis in patient with cirrhosis and refractory ascites: a randomized trial. J Hepatol 25: $135-144$

Lind CD, Malisch TW, Chong WK et al (1994) Incidence of shunt occlusion or stenosis following transjugular intrahepatic portosystemic shunt placement. Gastroenterology 106:1277-1283

Merli M, Salerno F, Riggio O et al (1998) Transjugular intrahepatic portosystemic shunt versus endoscopic sclerotherapy for the prevention of variceal bleeding in cirrhosis: a randomized multicenter trial. Hepatology 27:40-45

Nazarian GK, Ferral H, Castaneda-Zuniga WR et al (1994) Development of stenoses in transjugular intrahepatic portosystemic shunts. Radiology 192:231-234

Nicolao F, Efrati C, Masini A et al (2003) Role of determination of partial pressure of ammonia in cirrhotic patients with and without hepatic encephalopathy. J Hepatol 38:441-446

Nolte W, Wiltfang J, Schindler C et al (1998) Portosystemic hepatic encephalopathy after transjugular intrahepatic portosystemic shunt in patients with cirrhosis: clinical, laboratory, psychometric and electroencephalographic investigations. Hepatology 28:1215-1225

Pocock SJ (1976) The combination of randomized and historical controls in clinical trials. J Chronic Dis 29:175-188, U.S

Rajiv J, Dharmesh K (2004) Reversal of diuretic-induced hepatic encephalopathy with infusion of albumin but not colloid. Clin Sci 106:467474

Riggio O, Merli M, Pedretti R et al (1996) Hepatic encephalopathy after transjugular intrahepatic portosystemic shunt. Incidence and risk factors. Dig Dis Sci 41:578-584

Riggio O, Masini A, Efrati C et al (2005) Pharmacological prophylaxis of hepatic encephaolpathy after transjugular intrahepatic portosystemic shunt: a randomized controlled study. J Hepatol 42:674-679

Riggio O, Angeloni S, Salvatori FM et al (2008) Incidence, natural history, and risk factors of hepatic encephalopathy after transjugular intrahepatic portosystemic shunt with polytetrafluoroethylenecovered stent grafts. Am J Gastroenterol 103:2738-2746

Riggio O, Ridola L, Angeloni S et al (2010) Clinical efficacy of transjugular intrahepatic portosystemic shunt created with covered stents with different diameters: results of a randomized controlled trial. J Hepatol 53(2):267-272

Rossi P, Salvatori F, Fanelli F et al (2004) Polytetrafluoroethylenecovered nitinol stent-graft for transjugular intrahepatic portosystemic shunt creation: 3-year experience. Radiology 231: $820-830$

Rossle M, Deibert P, Haag K et al (1997) Randomised trial of transjugular intrahepatic portosystemic shunt versus endoscopy plus propranolol forprevention of variceal rebleeding. Lancet 349:1043-1049

Rossle M, Ochs A, Gulberg V et al (2000) A comparison of paracentesis and transjugular intrahepatic portosystemic shunting in patients with ascites. N Engl J Med 342:1701-1707

Salerno F, Merli M, Riggio O et al (2004) Randomized controlled study of TIPS versus paracentesis plus albumin in cirrhosis with severe ascites. Hepatology 40:629-635

Salerno F, Gerbes A, Ginès P et al (2007) Diagnosis, prevention and treatment of hepatorenal syndrome in cirrhosis. Gut 56:1310-1318

Sampietro G, Rossi P, Di Marco P (1998) Use of a laryngeal mask in transjugular intrahepatic portosystemic shunt procedures. J Vasc Interv Radiol 9:169

Sanjal AJ, Freedman A, Shiffman ML et al (1994) Portosystemic encephalopathy after transjugular intrahepatic portosystemic shunt: results of a prospective controlled study. Hepatology 20:46-55

Sanjal AJ, Freedman AM, Luketic VA et al (1996) Transjugular intrahepatic portosystemic shunts for patients with active variceal 
hemorrhage unresponsive to sclerotherapy. Gastroenterology 111: $138-146$

Sanyal AJ, Genning C, Reddy KR et al (2003) The North American study for the treatment of refractory ascites. Gastroenterology 124:634 641

Simón-Talero M, García-Martínez R, Torrens M et al (2013) Effects of intravenous albumin in patients with cirrhosis and episodic hepatic encephalopathy: a randomized double-blind study. J Hepatol 59(6): 1184-1192
Somberg KA, Riegler JL, LaBerge JM et al (1995) Hepatic encephalopathy after transjugular intrahepatic portosystemic shunt: incidence and risk factors. Am J Gastroenterol 90:549-555

Sort P, Navasa M, Arroyo Vet al (1999) Effect of intravenous albumin on renal impairment and mortality in patients with cirrhosis and spontaneous bacterial peritonitis. N Engl J Med 341:403-409

Zuckerman DA, Darcy MD, Bocchini TP et al (1997) Encephalopathy after transjugular intrahepatic portosystemic shunt: analysis and incidence of potential risk factors. Am J Radiol 169:1727-1731 